\title{
Open Government Data: The German Government is Moving
}

\author{
Chang Liu ${ }^{1, a}$, Xuan Liu ${ }^{2, b, *}$, Xiaohong $\mathrm{Li}^{3}$, Wenyan Liu ${ }^{4}$, Changyan Yan ${ }^{5}$, \\ Qing $L i^{6}$ \\ $1,2,3,4,5,6$ Library of Huazhong University of Science and Technology Wuhan, Hubei, China \\ a liuchang2008@hust.edu.cn, ${ }^{\mathrm{b}}$ liuxuan@hust.edu.cn
}

Keywords: Government Data, Germen, G7, Open Data, Platform

\begin{abstract}
Based on the introduction of open government data movement in Germany, this article selected the open data platforms of German as a research object to explore the better construction strategies by recognizing current status of G7 Open Government Data platforms through investigation, thus to provide suggestions for the development of Open government data platforms in China.
\end{abstract}

\section{Introduction}

On June 17, 2013, the Group of Eight (referred to as G8) Summit held in the United Kingdom, including the United States, Britain, France, Germany, Italy, Canada, Japan and Russia. The G8 leaders discussed Open Data together and signed the Open Data Charter (hereinafter referred to as the "Charter"), five basic principles for Open Data were established: in the case of standards, mutual recognition of open data, the quality and data of open data; Universality of open data; Open data is the responsibility and obligation of each government; Open data is for innovative services. The development of the "Charter" has been hailed as a landmark milestone in the process of opening data, and it has become the basis and gist for the implementation of open government data in G8 countries and other countries and regions of the world.

Since the signing of the "Charter" by the G8 countries, the G8 governments have made continuous progress and notable achievement in the Open Government Data. The Open Data Barometer, published by the World Wide Web Foundation, has released four global assessment reports since 2013. As a result of Russia's withdrawal in 2014, the G8 group evolved into a G7 group. The score performance of G7 Governments for Open Data Platform in the Open Data Barometer from 2013 to 2016 has been shown in Figure 1 [1].

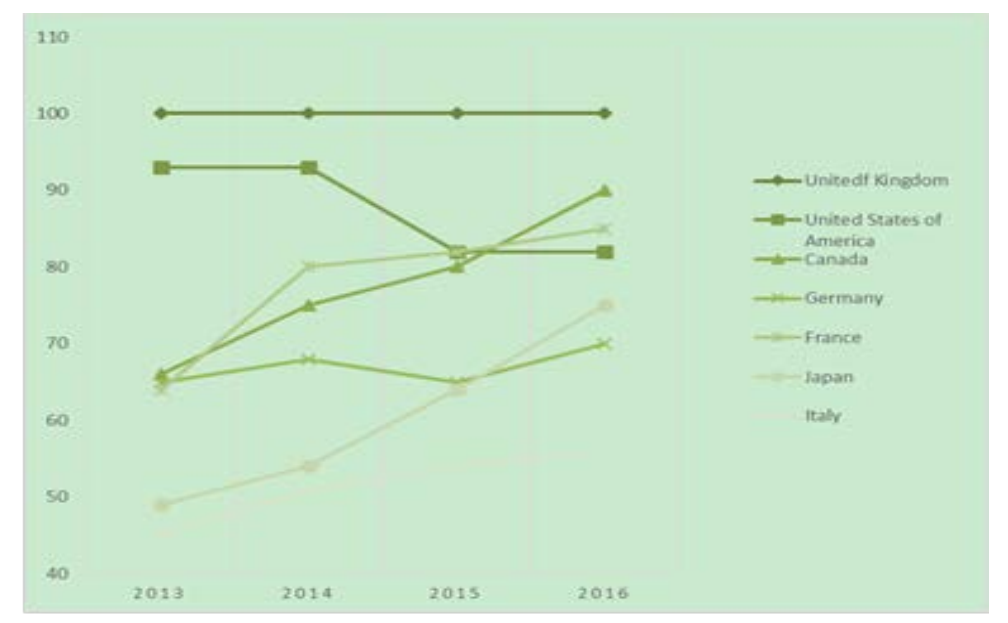

Fig. 1. The Scoring Performance of G7 Open Government Data Platforms in the Open Data Barometer Global Report(2013-2016)

Overall, the openness of G7 Group national government data was very high, which were ranked 
in the world's top 20 in the Open Data Barometer[2]. In recent years, Germany has shown a steady and excellent performance in open government data. The German government has not only formulated a policy agenda and action measures, but also built a Unified website GovData for open government data. As Germany belongs to civil law country, which has some similarities with China in the government planning and laws and regulations.

\section{Overview of Open Government Data in Germany}

\subsection{Action Plan}

In the past few years, based on the efforts made at the federal level, German government has also worked with the National IT Planning Committee and the Bundesland to further open data as a federal obligation and national policy. The action plan has taken into account for promoting the relevant outcomes of open data and the framework of open government data, including:

"Network-based and Transparent Management"—the 17th Government Planning Project

"Promoting Open Government Data"— key project of IT Planning Committee

Developing and upgrading the government data original portal site model (http://www.govdata.de) across departments and levels.

\subsection{Laws and Regulations}

In order to be legitimate, the federal government has been setting up a series of "Open Data Regulations" to unify the government-administered data, to read and parse kinds of basic data in machine-readable formats, with open licenses or other relevant hardware and software tools.

During the 17th tenure of the Bundesrath, relevant laws and regulations have been developed for the collection and storage of digital spatial data (GeoZG) access/channel and Bundesland space data (GeoNutzV). So the federal government has taken a crucial first step towards opening up government data in the direction of open data. On this basis, these laws and regulations would further serve the implementation and application of the national action plan for the open management data. For example, the federal government, in accordance with the regulations of EGOV Article 12, paragraph 2, developed a Geonutzv-user-oriented application on a simple and open basis, and found many applications related to the management data. In order to enable government data to be applied across borders, regions and industries, the use of standards and regulations needs to be adjusted to meet internationally recognized "Open Data Standards" requirements. The transmission and application of environmental information data should be based on UIG Article 10 (environmental information directive 2003/4/EG Article 7 and the Aarhus Convention of the United Nations Economic Commission for Europe, Article 5), with a positive attitude and posture, to effectively comply with the obligations, and try best to implement and always check its implementation results.

\subsection{Set up open data organization in the Federation}

The Federal Ministry of the Interior has established a dedicated open data agency, which undertakes overall and coordinated work in the federal government's regulation and control data in the portal "GoveData" applications across the federal, prefecture and municipal areas, in order to further implement the "positive-open-data" principle within the responsibilities of the federal government's regulatory body. In addition, all departments has nominated "open-data-coordinators" at the end of the third quarter of the year after 2014. Following this responsibility and commitment, the coordinator would be responsible for the coordination of collection, storage, publication of open data and open data applications for all departments, business areas, industries and individuals. 


\section{Introduction to the GovData Portal of the German Government's Open Data Platform}

\subsection{Overview of GovData Portal Site}

In accordance with the purposes of the G8 Charter, Germany, like the other seven countries, has developed its own national action plan after the Summit[3], which took a variety of initiatives from a number of dimensions, such as law, technology, information infrastructure and applications, to facilitate the opening of data by the federal government and local governments at all levels[4]. The official open data portal (http://www.govdata.de) [5] has been subsequently built for data management, querying and reuse in Germany. The collected, consolidated, filtered and purged government data would be grouped in six columns of data, documents, applications, letters, blogs, and standard specifications [6], GovData portals open these data to the public and provide convenient, versatile search and navigation capabilities for organizations, groups and individuals to find, discover, and apply the desired target open data resources, thus to make great progress and achievements in sharing the application of open data.

\section{2 “DCAT-AP.DE” Adopted by GovData Portal}

The application of open data can not be separated from the high quality metadata support, DCAT (Data Catalog Vocabulary) was the rich party recommendation standard of the government open metadata data [7]. DCAT was drafted by the National University of Ireland and was revised by the WC3 E-Government Professional Committee, which was finally released by the WC3 Open Government Data Working Committee. According to the international general DCAT standard, the European Union and the United Kingdom proposed the open data metadata scheme-"The DCAT of the European Union data portal and its application outline", namely DCAT-AP (Application Profile Fordata Portals in Europa). On the basis of this outline, the German government, through its work in the areas of normalization, standardization, semantization and machine-readable, has greatly improved the quality of metadata, facilitated the interoperability of local government data among the corresponding directories, and laid a good foundation for further development of metadata for the theme application industry and domain.

\subsection{Directory Paths and Navigation}

According to the principles and standards of open data, it would be conducive to collection, integration, retrieval, analysis, research, reuse and other various functions of open data. The data set is categorized to clearly and intuitively provide users with the following 14 main application group types: population, education and science, geography, geology and spatial data, law and justice, health, infrastructure living and life, culture, leisure, sports and tourism, public administration, budget and taxation, politics and elections, society, transportation and traffic, environment and climate, consumer protection, economics and employment [8]. In these open theme application group types, the German government has stored, collected and prepared a large number of data sets to lay a solid foundation for the open data work.

\subsection{Latest Entries}

In order to facilitate users to timely search and timely access to the latest information, as well as the development of open data, the GovData portal creates a latest update column, including the latest data sets, the latest documents, the latest blog, and several sub columns, to provide up-to-date legal compliance data for citizens and users at any time.

\section{The Revelation of the Open Data Movement of the German Government to China}

\subsection{Cooperation Between States and Citizens, Country and Region, National and International}

In order to promote and implement all the measures of national action plan and to achieve the highest efficiency of its application, effective cooperation in the field of open government data 
should be strengthened with the citizens, economic circles, scientific and technological sectors, the media and regulatory bodies. China has begun to open data on administrative agencies and continues to promote cooperation at all levels. At the same time, the provincial and municipal local government portals have implemented the "open-data-strategy", gradually open the directory of existing data. Another objective of the government is to continue to seek exchanges and cooperation with civil society organizations at the national level. At the international level, multinational talks and cooperation will be continued. In cooperation with citizens, it is possible to set up relevant open day and organize more specific and special theme competitions. In these activities, civil society groups from the relevant parties, economic circles, media, scientific and technological sectors and other important application developers should be introduced to involve in the active participation to closely monitor the government's open data and its wide application.

\subsection{Focus on Designing Participation Programs for Open Data Users}

Open data is not the only and ultimate goal, and its ultimate goal is to apply and achieve benefits. The Government should therefore establish a regular dialogue mechanism, in particular to maintain close contacts, share experiences and create results with civil society groups, the economic and social sectors, the media and the scientific community [9].

\subsection{Provide Important and Valuable Data Services for Innovation}

To serve innovation and to contribute to social and economic development, government should focus on the quality, value and effectiveness of open data, in particular on the promotion of innovation and the provision of valuable services to the public. Therefore, the federal government and the user accelerated the process of open data and subject applications in the following important industries through careful research jointly, in the context of open data, some valuable data will be recommended to users at the highest possible level of priority, such as transportation and mobile communications, the energy revolution, climate change and environmental protection, demographic changes, the network and the technological revolution of infrastructure, public revenue and expenditure.

\section{Acknowledgement}

In this paper, the research was sponsored by the Research Fund Investigation Program of Hubei Academic Library Committee and China Economic Information Network (Project No. 2016ZJYB01) .

\section{References}

[1] Open Data Barometer Global Report [EB/OL].[2018-03-03]http://opendatabarometer.org/ a) report.

[2] Open Data Readiness Assessment[EB/OL].[2018-03-05]http://opendatatoolkit.worldbank.org/

a) en/odra.html.

[3] Global Open Data Index [EB/OL].[2018-03-06]https://index.okfn.org.

a) Open Government Data

Survey[EB/OL].[2018-03-06]https://publicadministration.un.org/en/.

[4] Govdata.[EB/OL].[2018-04-15]http://www.govdata.de

[5] Huang Ruhua, Li Nan.The research fo licenses for open government data. Library and Information Service, vol. 60, issue 13, pp. 5-12, 2016. 
[6] DCAT-AP.DE.[EB/OL].[2018-04-15]https://www.govdata.de/web/guest/neues/-/blogs/dcat-apde-die-entwicklung-geht-weiter

[7] Cao Yujia, Government Open Data Survival Status: Investigation Report on 19 Local Governments. Library and Information Service, vol. 60, issue 14, pp.94-101, 2016.

[8] Zheng Lei, Study on Open Government Data:Definitions, Factors and Interactions. Chinese Public Administration, Chinese Public Administration, vol. 11, pp13-18, 2015. 